\title{
Semiconductor guidestar laser for astronomy, space, and laser communications: prototype design and expected performance
}

Céline d'Orgeville, Gregory J. Fetzer, Steven Floyd, Luke Hill, Steve Rako, et al.

Céline d'Orgeville, Gregory J. Fetzer, Steven Floyd, Luke Hill, Steve Rako, Nathan Woody, . Sandalphon, Francis Bennet, Antonin Bouchez, Yue Gao, Michael Goodwin, Andrew Lambert, James Mason, Francois Rigaut, Stuart Ryder, Daniel Shaddock, Robert Sharp, "Semiconductor guidestar laser for astronomy, space, and laser communications: prototype design and expected performance," Proc. SPIE 10703, Adaptive Optics Systems VI, 107030T (10 July 2018); doi: 10.1117/12.2312405

Event: SPIE Astronomical Telescopes + Instrumentation, 2018, Austin, Texas, United States 


\title{
Semiconductor Guidestar Laser for Astronomy, Space, and Laser Communications: Prototype Design and Expected Performance
}

Céline d'Orgeville ${ }^{* a}$, Gregory J. Fetzer ${ }^{\mathrm{b}}$, Steven Floyd ${ }^{\mathrm{b}}$, Luke Hill ${ }^{\mathrm{b}}$, Steve Rako ${ }^{\mathrm{b}}$, Nathan Woody ${ }^{\mathrm{b}}$, Sandalphon ${ }^{\mathrm{c}}$, Francis Bennet ${ }^{\mathrm{a}}$, Antonin Bouchez ${ }^{\mathrm{d}}$, Yue Gao ${ }^{\mathrm{e}}$, Michael Goodwin ${ }^{\mathrm{f}}$, Andrew Lambert ${ }^{\mathrm{g}}$, James Mason ${ }^{\mathrm{h}}$, Francois Rigaut ${ }^{\mathrm{a}}$, Stuart Ryder ${ }^{\mathrm{f}}$, Daniel Shaddock ${ }^{\mathrm{i}}$, Robert Sharp ${ }^{\mathrm{a}}$

${ }^{a}$ Research School of Astronomy and Astrophysics, Australian National University, Canberra, Australia; ${ }^{b}$ Areté Associates, Longmont, Colorado, USA; ${ }^{\mathrm{c} C i n n a b a r}$ Optics, Louisville, Colorado,

USA; ${ }^{\mathrm{d}}$ GMTO Corporation, Pasadena, California, USA; ${ }^{\mathrm{e}}$ EOS Space Systems, Queenbeyan, Australia; ${ }^{\mathrm{f} A A O}$, Sydney, Australia; ${ }^{\mathrm{g} U N S W}$, Sydney, Australia; ${ }^{\mathrm{h}}$ Lockheed Martin Space Systems, Advanced Instrumentation Center, Palo Alto, California, USA; ${ }^{i}$ Research School of Physics and Engineering, Australian National University, Canberra, Australia

\begin{abstract}
A new type of sodium guidestar laser based on semiconductor laser technology is being developed by the astronomy, space, and laser communication communities in Australia and the United States, in partnership with laser manufacturer Areté Associates. Funding has been secured from the Australian Research Council and the Australian National University, with support from academic (UNSW) and industry partners (AAO, GMTO, EOS, Lockheed Martin). The consortium aims to develop a full scale prototype of the Semiconductor Guidestar Laser. The laser, to be delivered in 2019, will be initially installed on the EOS Satellite and Debris Tracking Station 1.8m telescope at Mount Stromlo Observatory where it will be thoroughly tested, on sky and in real operation conditions. This will be the first time that a Laser Guide Star is created in Australian skies. We present the project motivation and objectives, laser development and test plans, and the preliminary test results obtained to date.
\end{abstract}

Keywords: Sodium laser guide star adaptive optics, semiconductor guidestar laser, optically-pumped semiconductor laser, space situational awareness, laser communications

\section{SEMICONDUCTOR GUIDESTAR LASER PROJECT}

The context and motivation for the Semiconductor Guidestar Laser project was presented in details two years ago in the proceedings for the 2016 SPIE Adaptive Optics conference ${ }^{1}$. At the time, funding was being sought to support the development of this $4^{\text {th }}$ generation of sodium guidestar laser systems, to be based on semiconductor laser technology. Since then, funding has been secured from an Australian Research Council (ARC) Linkage Infrastructure Equipment and Facilities (LIEF) grant and an Australian National University (ANU) Major Equipment grant, with support from academic (University of New South Wales) and industry partners (Australian Astronomical Observatory, Giant Magellan Telescope Organization, EOS Space Systems, and Lockheed Martin Space Systems).

*celine.dorgeville@anu.edu.au; phone +61 26125 6374; fax +61 26125 0233; rsaa.anu.edu.au

Adaptive Optics Systems VI, edited by Laird M. Close, Laura Schreiber,

Dirk Schmidt, Proc. of SPIE Vol. 10703, 107030T - @ 2018 SPIE

CCC code: $0277-786 \mathrm{X} / 18 / \$ 18 \cdot$ doi: $10.1117 / 12.2312405$ 
The Australian National University is leading the effort to develop a full scale prototype of the Semiconductor Guidestar Laser which will be tested next year (2019) on the EOS laser tracking station $1.8 \mathrm{~m}$ telescope at Mount Stromlo Observatory near Canberra, Australia. The consortium aims to demonstrate that semiconductor laser technology is suitable to meet the demanding requirements of sodium Laser Guide Star Adaptive Optics (LGS AO) systems for astronomy, space surveillance, and laser communication applications on telescope of all sizes.

Semiconductor Guidestar Laser technology offers the promises of an affordable, efficient, and low SWaP (Size, Weight and Power) alternative to the current sole-source commercial system available for sodium guidestar laser applications, i.e. the Toptica 20/2 SodiumStar ${ }^{2}$.

The ANU has contracted US laser vendor Areté Associates to design and fabricate the Semiconductor Guidestar Laser prototype. Laboratory work started in September 2017 to investigate 1178nm and $589 \mathrm{~nm}$ semiconductor laser performance in a laboratory environment before converging on an engineered design for the laser head and laser electronic enclosure. To date, two reviews have taken place at Areté Associates' headquarters in Longmont, Colorado: a System Requirements Review was held in April 2018, and a System Design Review in June 2018, both showing promising performance results and significant design progress towards the final engineered prototype.

The laser prototype requirements and expected performance are presented in section 2; laboratory results to date are presented in section 3; and the prototype system design is presented in section 4.

\section{PROTOTYPE REQUIREMENTS}

Table 1 shows the target performance requirements for the semiconductor guidestar laser prototype. The projected laser power typically required per LGS from a continuous wave (CW) single frequency sodium guidestar laser for year-round LGS AO observations on large telescopes at good astronomical sites is on the order of 10-20W at the sodium D2 line ${ }^{3-5}$. The power planned for this development is 8-10 W in resonance with the $\mathrm{D}_{2 \mathrm{a}}$ transition. An enhancement of sodium return signal is achieved if light resonant with the sodium $\mathrm{D}_{2 \mathrm{~b}}$ transition (shifted by $1.77 \mathrm{GHz}$ relative to $\mathrm{D}_{2 \mathrm{a}}$ ) is broadcast simultaneously and overlaps the beam of the first wavelength. The second wavelength overcomes trapping of atoms in hyperfine levels of the ground state of the $\mathrm{D}_{2 b}$ transition by re-pumping them to the total available population. Thus a secondary wavelength is included (Table 1) with $\sim 10 \%$ of the $\mathrm{D}_{2 \mathrm{a}}$ power - the power estimated to provide for significant enhancement.

The laser must have a narrow linewidth in order to efficiently excite the sodium atoms. The laser must also be continuously tunable over the width of each $\mathrm{D}_{2}$ transition $(\sim 1 \mathrm{GHz})$ to allow the laser emission to be locked to the transition. Additionally, it is desirable to have the ability to tune off of the sodium resonance in order to obtain an estimate of the 'Rayleigh background' during LGS AO operations. The laser output must have good beam quality to enable small spot sizes, and well-defined polarization to optimize the return. 
Table 1: Semiconductor Guidestar Laser prototype contractual performance requirements, goals, and expected performance based on laboratory results obtained to date (7 June 2018).

\begin{tabular}{|c|c|c|}
\hline Characteristic & Requirement (Goal) & Expected \\
\hline $\begin{array}{l}\text { Primary Wavelength and } \\
\text { Power }\end{array}$ & $\begin{array}{l}\geq 5 \mathrm{~W} \text { (goal: } 25 \mathrm{~W}) \text { locked to } \\
\mathrm{Na}\left(\mathrm{D}_{2 \mathrm{a}}\right) \sim 589 \mathrm{~nm}\end{array}$ & $\begin{array}{l}\text { 8-12 W locked to } \mathrm{Na}\left(\mathrm{D}_{2 \mathrm{a}}\right) \sim 589 \\
\mathrm{~nm}\end{array}$ \\
\hline $\begin{array}{l}\text { Secondary Wavelength and } \\
\text { Power }\end{array}$ & $\begin{array}{l}\geq 0 \mathrm{~W} \text { (goal: } 10 \% \text { of power at } \\
\text { primary wavelength) in } \\
\text { resonance with } \mathrm{Na}(\mathrm{D} 2 \mathrm{~b})\end{array}$ & $\begin{array}{l}10 \% \text { of power at primary } \\
\text { wavelength }\end{array}$ \\
\hline Waveform & Continuous Wave & Continuous Wave \\
\hline Linewidth & $\leq 50 \mathrm{MHz}$ (goal: 10MHz) & $10 \mathrm{MHz}$ \\
\hline Fine Tuning & $\begin{array}{l}\geq 1 \mathrm{GHz} \text { (goal: } 3 \mathrm{GHz} \text {, } \\
\text { continuous }\end{array}$ & $\geq 1 \mathrm{GHz}$ \\
\hline Gross Tuning & $\begin{array}{l}\geq 5 \mathrm{GHz} \text {, does not need to be } \\
\text { continuous }\end{array}$ & $\geq 5 \mathrm{GHz}$ \\
\hline Beam Quality & $\mathrm{M}^{2} \leq 1.5\left(\right.$ goal: $\left.\mathrm{M}^{2} \leq 1.2\right)$ & $\mathrm{M}^{2} \leq 1.5$ \\
\hline Polarization & $\begin{array}{l}\text { Well defined polarization, } \\
\text { contrast ratio } \geq 20 \text { (goal: } 40)\end{array}$ & Linear polarization \\
\hline
\end{tabular}

The expected $\sim 10 \mathrm{~W}$ system represents a compromise between cost and performance. This laser will be a brassboard device that provides the first demonstration of a semiconductor guidestar laser in an observatory while keeping costs to a reasonable level.

\section{LABORATORY RESULTS TO DATE}

Areté's brassboard laser development plan uses a two-pronged approach to minimize risk and maintain schedule. As shown in Figure 1 the Tech Demonstrator portion of the program consists of laboratory based laser experimentation and mitigates performance risk by allowing agile, flexible experiments where factors influencing laser performance are separable. Results of these experiments are fed into the brassboard design throughout the program which is conducted in parallel. Performing the program in this fashion allows Areté to meet the desired development timeframe.

\begin{tabular}{|c|c|c|c|c|c|c|c|c|c|c|c|c|c|c|c|c|}
\hline & \multicolumn{4}{|c|}{2017} & \multicolumn{4}{|c|}{2018} & \multicolumn{4}{|c|}{2019} & \multicolumn{4}{|c|}{2020} \\
\hline Task & Q1 & Q2 & Q3 & Q4 & Q1 & Q2 & Q3 & Q4 & Q1 & Q2 & Q3 & Q4 & Q1 & Q2 & Q3 & Q4 \\
\hline \multicolumn{17}{|l|}{ Australian National University } \\
\hline Tech Demonstrator & & & & & & & & & \multicolumn{4}{|c|}{$8-12 W D_{2 a}+D_{2 b}$} & & & & \\
\hline \multicolumn{17}{|l|}{ Brassboard Development } \\
\hline \multicolumn{17}{|l|}{ On Sky Demonstration } \\
\hline & & & & & & & & & & & & & & & & \\
\hline
\end{tabular}

Figure 1: Semiconductor Guidestar Laser Program Schedule (Prototype Phase).

As part of the Tech Demonstrator portion of the program Areté constructed both I-cavity and V-cavity lasers. The configuration of each cavity is shown in Figure 2 both schematically and pictorially. The 
I-cavity laser was used primarily to characterize gain mirror performance as a function of configuration variables such as gain mirror plate temperature, pump laser powers and cavity parameters such as output coupler mirror transmissivity, radius of curvature and cavity length. All of these experiments were conducted with the laser operating in the fundamental near $1178 \mathrm{~nm}$. The Vcavity configuration was used to characterize performance in both the fundamental and for frequency doubled operation near 589nm.

A number of laser design parameters were derived through laser cavity modeling and experimental performance demonstrations. While performance has been impressive to date, significant opportunity exists to continue to improve all facets of the design to arrive at a fieldable VECSEL guide star laser.

While a variety of $808 \mathrm{~nm}$ pump lasers have been employed to date, the most recent work has been conducted with a 200 micrometer fiber coupled array of diodes capable of $70 \mathrm{~W}$ of output power at an electrical to optical efficiency of approximately $40 \%$. Thermal management of the gain mirrors is accomplished through a combination of thermoelectric and water cooling.

When operating the lasers with intracavity elements we control the temperature of the birefringent filter and the etalon. The lithium triborate (LBO) Second Harmonic Generation (SHG) crystal is held at the phase matching temperature near $40^{\circ} \mathrm{C}$.
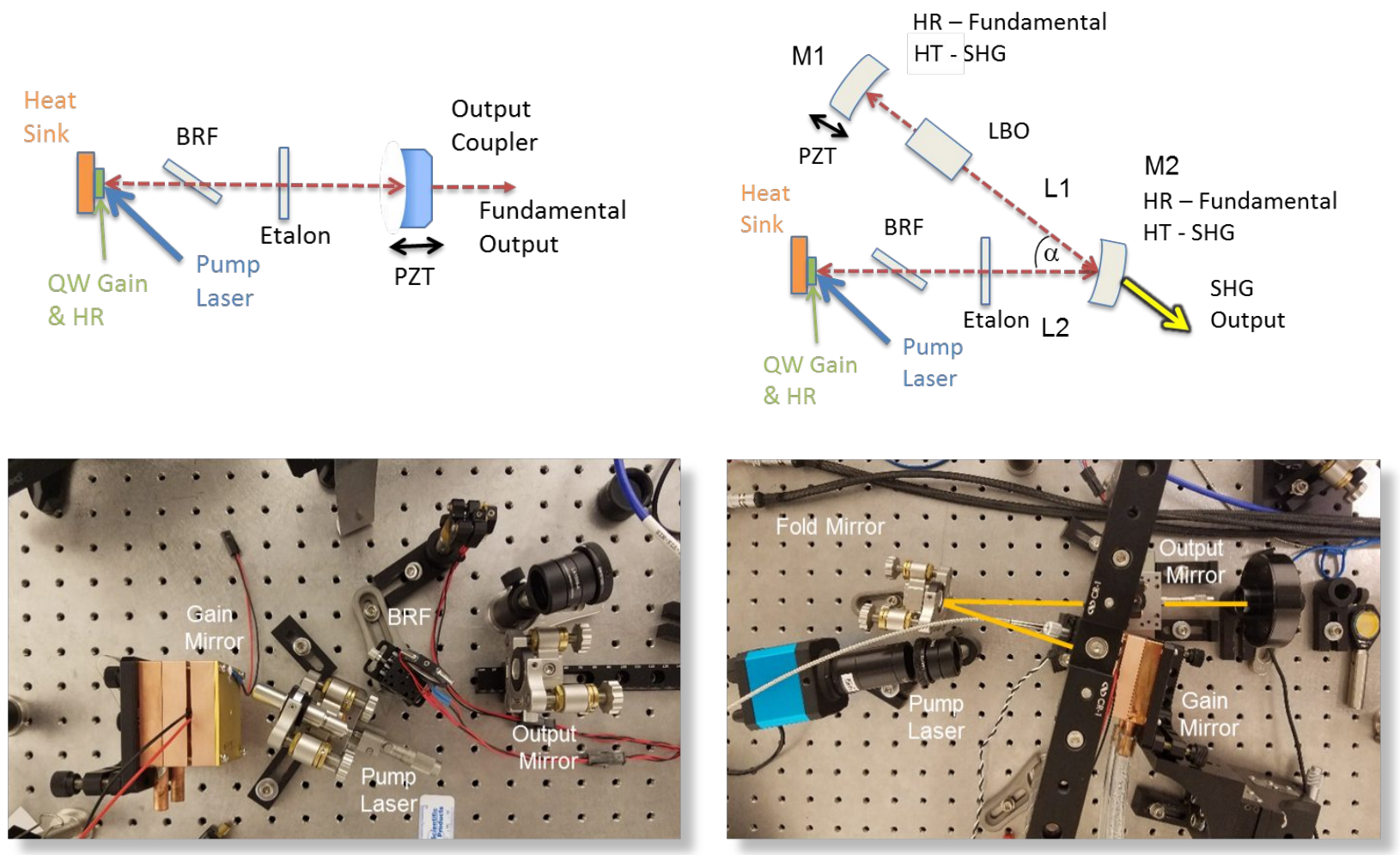

Figure 2: The top half of the figure contains cartoon schematics of the I-Cavity and V-Cavity. The bottom half of the figure is pictures of the I-Cavity and V-Cavity implementations that were used to support measurements presented herein. 




Figure 3: VECSEL output power near $1178 \mathrm{~nm}$ for three water chiller temperatures. No attempt to stabilize the output wavelength was made to achieve these results.

The I-cavity laser was used to characterize gain mirrors and determine cavity design parameters. Figure 3 illustrates the output power of an I-cavity laser as a function of pump power for three chiller temperatures. The laser was operating with no intracavity elements. Consequently, the output was spectrally broad and centered around $1175 \mathrm{~nm}$.

Higher powers can be achieved by operating the system at cooler temperatures. By reducing the chiller temperature and the TEC set point to $10^{\circ} \mathrm{C}$ we were able to achieve in excess of $20 \mathrm{~W}$ of infrared power from the laser. The onset of parasitic losses from the gain mirror occurs at higher pump powers when heat is more effectively removed.

The broadly tunable emission of VECSELs is well documented in the literature. An intracavity spectrally dispersive element such as a birefringent filter (BRF) can be used to tune the laser to the desired wavelength. Areté used a $5 \mathrm{~mm}$ thick quartz (BRF) oriented at the Bragg angle with respect to the beam to provide wavelength tunability and spectral narrowing of the output of the I Cavity VECSEL. 

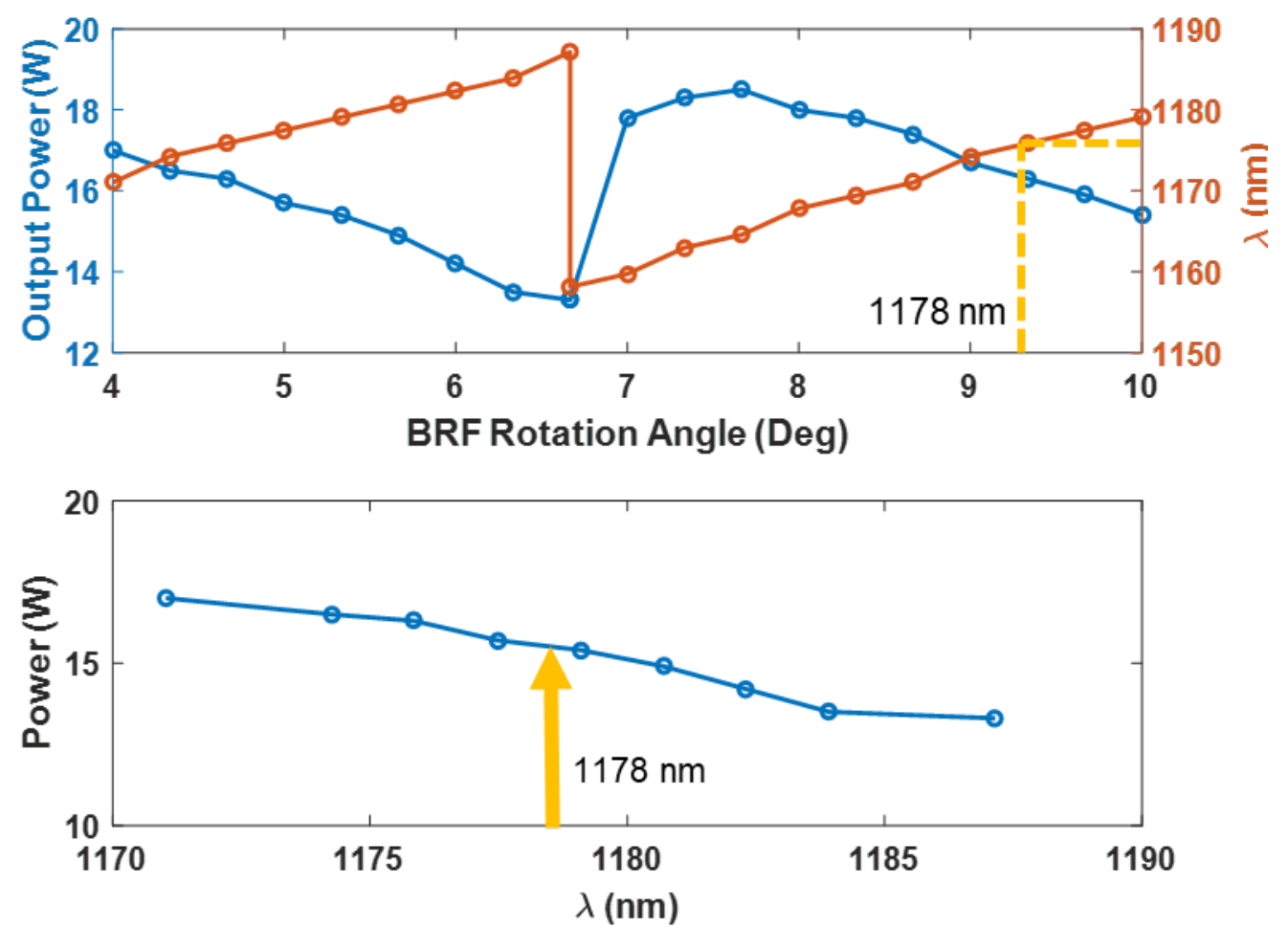

Figure 4: Operational characteristics of the I-Cavity laser with a birefringent crystal inserted at Brewster's angle into the cavity. The top pane plots the laser output power and wavelength as a function of the BRF rotation angle. The bottom pane plots the dependence of the laser output power as a function of wavelength.

Figure 4 provides the data collected as the BRF rotation angle was changed to effect tuning of the laser. The top pane represents raw data from the plot. The lower pane presents the output power plotted as a function of the wavelength achieved at various BRF angles. In the top pane the TEC and chiller temperature set points were $10^{\circ} \mathrm{C}$. The pump power was set at $55 \mathrm{~W}$. The blue line in Figure 4 plots the laser output power as a function of the BRF angle. The orange line plots the output wavelength as measured using a CCD spectrometer with a spectral resolution of $1.5 \mathrm{~nm}$. The sharp transition in the output power and the wavelength is expected and is a result of the BRF periodic spectral transmission characteristic with rotation angle. Insertion of the BRF into the cavity resulted in less than 5\% reduction in the maximum power achieved at this pump power. By rotating the BRF the laser is easily tuned to $1178 \mathrm{~nm}$. The output power is approximately $16 \mathrm{~W}$ at $1178 \mathrm{~nm}$. While not critical to the guide star application it is noteworthy that this particular mirror provides $15 \mathrm{~nm}$ of tuning with power exceeding $10 \mathrm{~W}$.

An important aspect of guidestar laser performance is stable narrow linewidth operation in resonance with the sodium transition. To achieve this characteristic, it is necessary to further restrict the spectral extent of lasing action in the VECSEL cavity. To accomplish this Areté, and others, use an etalon to greatly restrict the number of longitudinal modes that have sufficient gain to laser, to ideally a single mode. The homogeneously broadened gain medium of the gain mirror is relatively immune to spatial 
hole burning and single mode operation is readily achievable. Sufficient vibration and thermal stabilization is required to maintain mode hop free operation. Long term single frequency operation at a specific wavelength most often requires active feedback control. However, to demonstrate single frequency operation we inserted a BRF and an etalon into the V-cavity and operated the laser at $1178 \mathrm{~nm}$. Note that the output power of the laser was limited to several hundred milliwatts because both mirrors in the cavity were high reflectors at $1178 \mathrm{~nm}$.

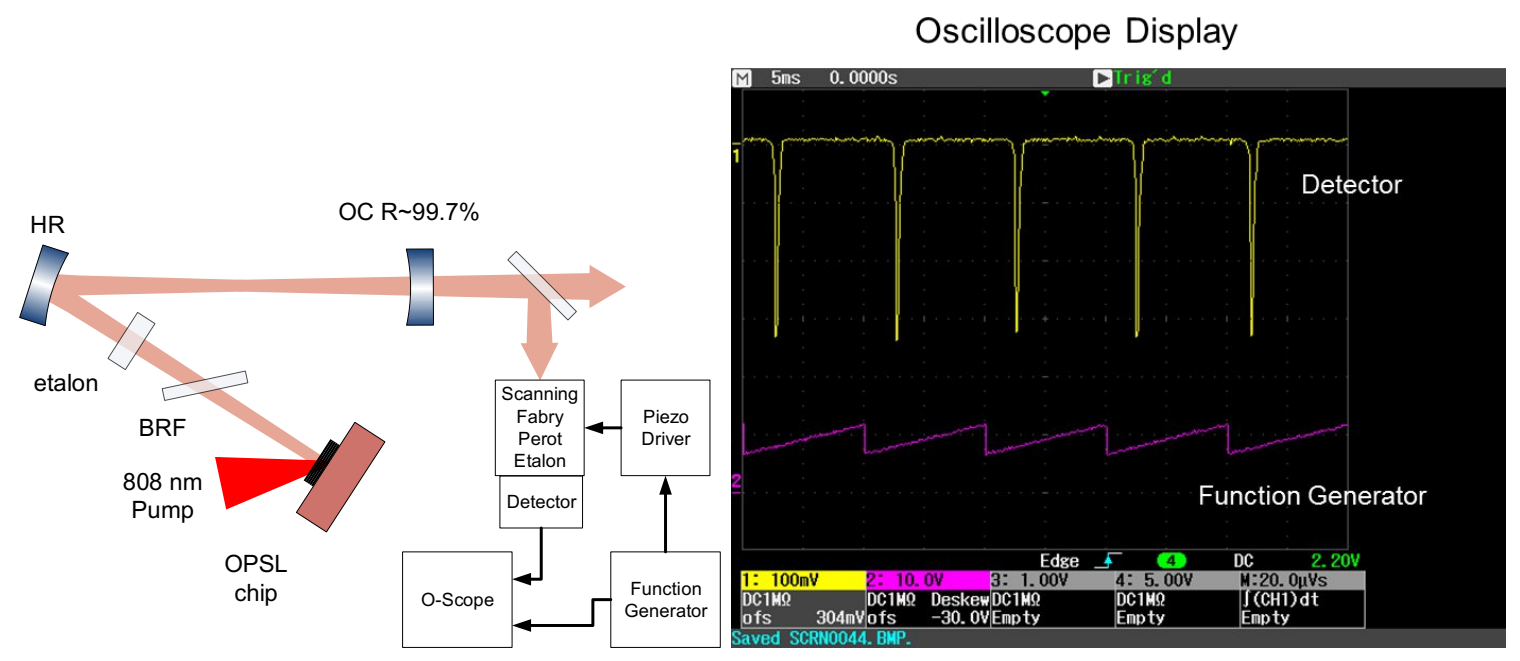

Figure 5: Example of single frequency operation of the V-cavity laser at the fundamental wavelength. The left pane shows an oscilloscope trace that was captured from the experiment shown in the right pane.

Figure 5 illustrates the configuration used to achieve and verify single frequency operation at $1178 \mathrm{~nm}$. As shown in the left pane a portion of the laser output is directed into a transmissive scanning Fabry Perot (FP) cavity. The fraction of the light transmitted by the etalon impinges on a photodetector. When the laser frequency coincides with the etalon resonance a strong signal appears on the photodetector. When out of resonance almost no light falls on the detector.

Under the condition that the etalon scan is greater than the longitudinal mode spacing of the laser the following is true. A function generator controls the scan width of the FP and serves to trigger the oscilloscope which monitors the signals out of the detector. If a laser is operating in many modes scanning the FP results in many impulses on the detector for each scan but when in single mode operation there should only be one burst per scan. That characteristic of single mode operation is seen in the right pane of the figure where only a single signal peak is seen in synchrony with the scan waveform of the function generator. The uniform spacing of the signal peaks verifies that the laser is operating at the same frequency for each scan.

We have not conducted rigorous measurement of the single frequency linewidth but in previous work using the same setup and slightly different VECSEL materials we were able to estimate the lasing linewidth at approximately $10 \mathrm{MHz}$. A similar experiment at 589nm is being conducted at this time. 
Our experience is that achieving single frequency operation in the fundamental is always a first step towards reaching that goal at harmonic wavelengths.

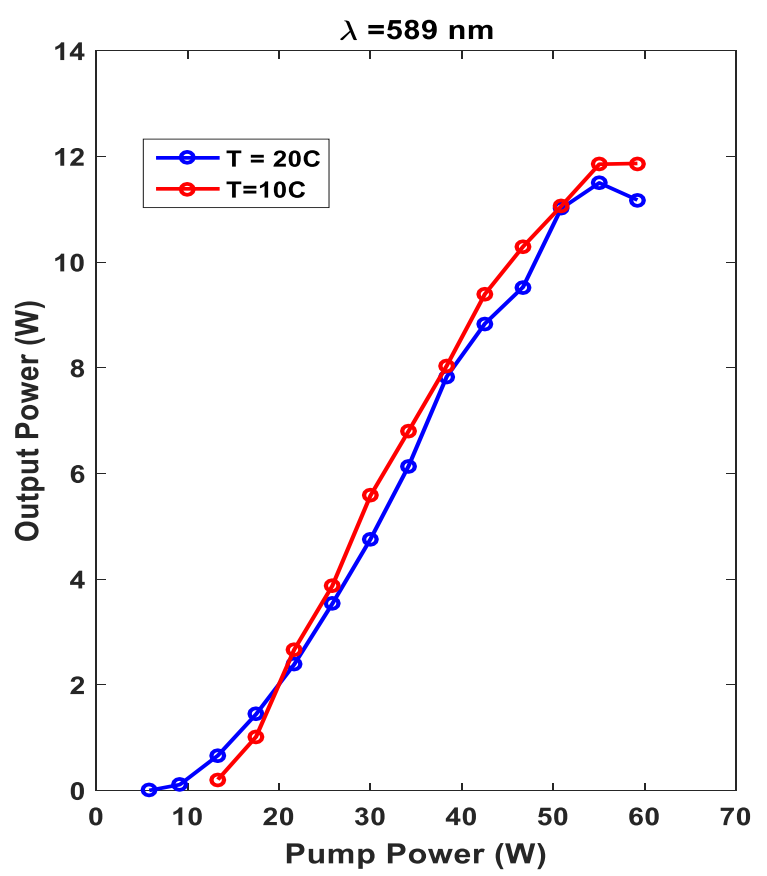

Figure 6: Output power of the V-cavity laser at $589 \mathrm{~nm}$ as a function of the pump power.

Areté used a LBO crystal in the V-cavity to generate output at $589 \mathrm{~nm}$. Our results to date are illustrated in Figure 6. Over $12 \mathrm{~W}$ of power at $589 \mathrm{~nm}$ was achieved. Optical-to-optical conversion efficiency at high pump powers is higher than $21 \%$. We observed that the factor limiting the output power was that the SHG crystal began to heat up due to absorption of the fundamental disturbing the temperature phase matching condition. We are taking measures to further optimize the output power by rectifying this issue through better thermal management of the crystal.

In another demonstration aimed at proving the resonance of the laser output with the sodium $\mathrm{D}_{2}$ transition, we directed a small portion of the laser output through a sodium cell heated to $70^{\circ} \mathrm{C}$. The path of the beam through the cell is clearly seen strongly fluorescing as the sodium interacts with the laser radiation (Figure 7).

By changing the etalon or BRF temperature we could move the wavelength of the laser off and back on the sodium resonance with high reliability and repeatability. The tuning was accomplished with little fluctuation in power. When off resonance there was no light coming from the cell other than a very small amount of scatter. Such tunability indicates that the laser is changing by more than $3 \mathrm{GHz}$. In some LGS AO applications, tuning off of the sodium resonance may enable calibration of the Rayleigh backscatter from the atmosphere in order to null out that return on the adaptive optics LGS wavefront sensors. 

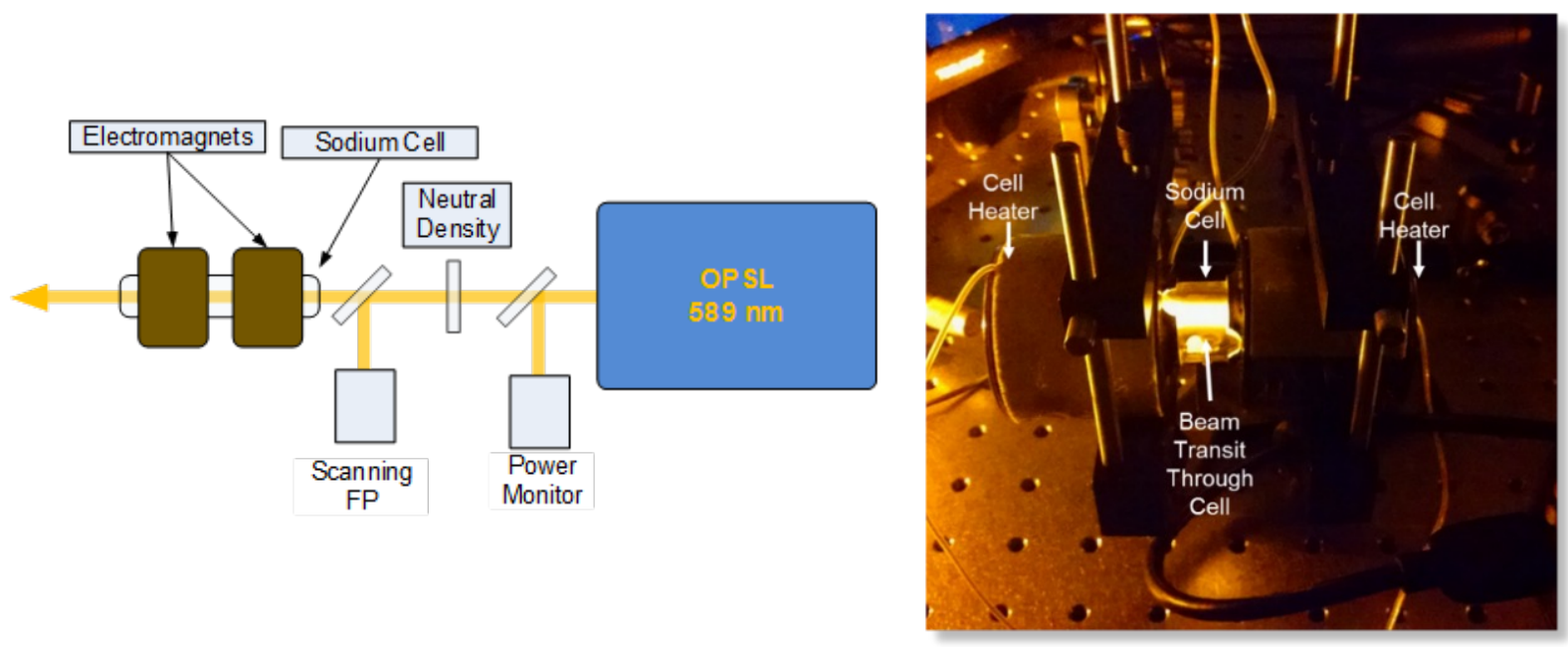

Figure 7: Example of V-cavity $589 \mathrm{~nm}$ beam transiting a sodium cell demonstrating resonance with the sodium $\mathrm{D}_{2}$ transition. Top pane - Diagram of experimental setup. Bottom panePhotograph of sodium emission from the sodium cell.

\section{PROTOTYPE SYSTEM DESIGN}

The semiconductor guidestar laser prototype consists of two assemblies. A 3D CAD rendering of the laser is shown in Figure 8. The approximate dimensions, weights and power requirements are included in the figure. The laser head has been designed to be very compact so that it can be mounted easily within the telescope structure and minimizes the difficulties of beam delivery to the launch telescope.

The system rack is small in comparison to those associated with traditional guide star lasers and provides power and control to the laser head. The laser head and the rack are interconnected by electronics and optical cable bundles that can extend to greater than $10 \mathrm{~m}$. The rack has modules to support optical diagnostics and wavelength control of the laser head. There are six dedicated shelves within the rack. The top rack provides power and Ethernet connections for a laptop for service or interim operation from the rack. The other shelves provide optical diagnostics, thermal management, power distribution, and computer control and includes communication to the outside world. The fundamental subsystems of the semiconductor guidestar laser prototype are shown in Figure 9.

The VECSEL cavity, the pump laser and the D2a/D2b module are housed in the laser head. The VECSEL cavity is a V-Cavity similar to that described above. The pump laser is a fiber coupled diode stack delivering approximately $120 \mathrm{~W}$ through a 200 micron fiber. The output of the V-cavity is directed into a module to generate both the D2a and D2b components necessary for efficient pumping of sodium in the mesosphere. Aretés approach to generating light resonant with the D2b line is to use a resonant electro-optic phase modulator to put approximately $10 \%$ of the power in a frequency sideband that is shifted by $1.71 \mathrm{GHz}$. Thus when the main beam is locked to the D2a line the sideband will coincide with the D2b line. 

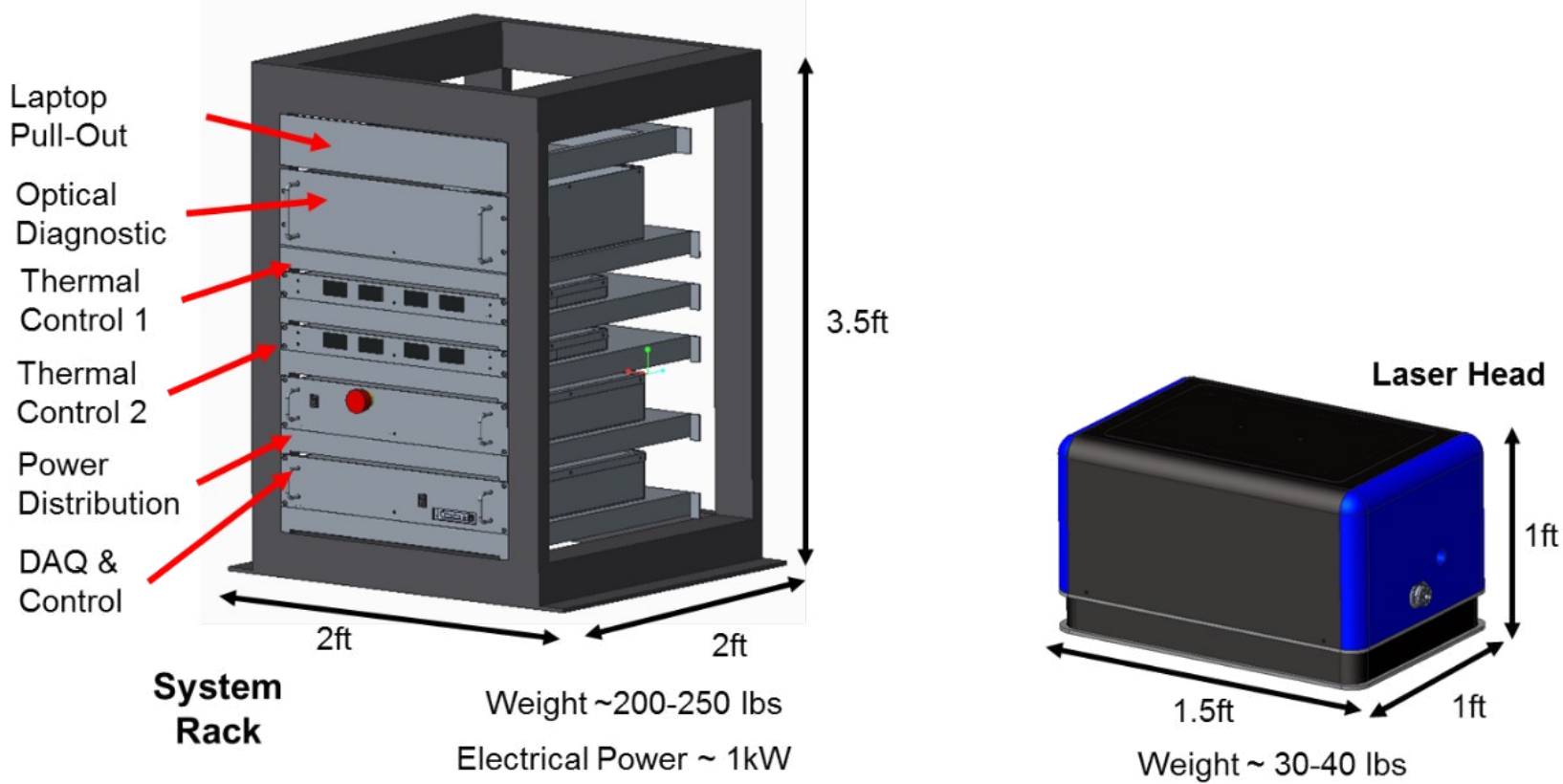

Figure 8: Semiconductor Guidestar Laser Prototype CAD Design: System Rack (left) and Laser Head (right).

A portion of the output from the $\mathrm{V}$-cavity is coupled into a fiber and directed to the Optical Diagnostics module where it is used to lock the laser wavelength to the sodium transition. Areté employs a PoundDrever-Hall locking technique to maintain the laser resonance with the sodium D2 transition. Additionally, the laser power and beam shape are monitored continuously as performance metrics.

A power distribution subsystem draws necessary power from the facility and internally delivers all required power forms within the rack and laser head.

The Data Acquisition and Control subsystem controls the system, monitors all functions and provides a user interface that provide the user remote access to operational parameters and the ability to log key performance metrics during operation.

Currently, the optical diagnostics, power distribution, thermal control, and DAQ subsystems of the System Rack are being assembled and tested. The laser head is still in design pending further exploration and development in the Tech Demonstrator. We anticipate completing the design early in the third quarter of 2018. Fabrication followed by integration and test will follow through the remainder of 2018. 
Guide Star Laser System

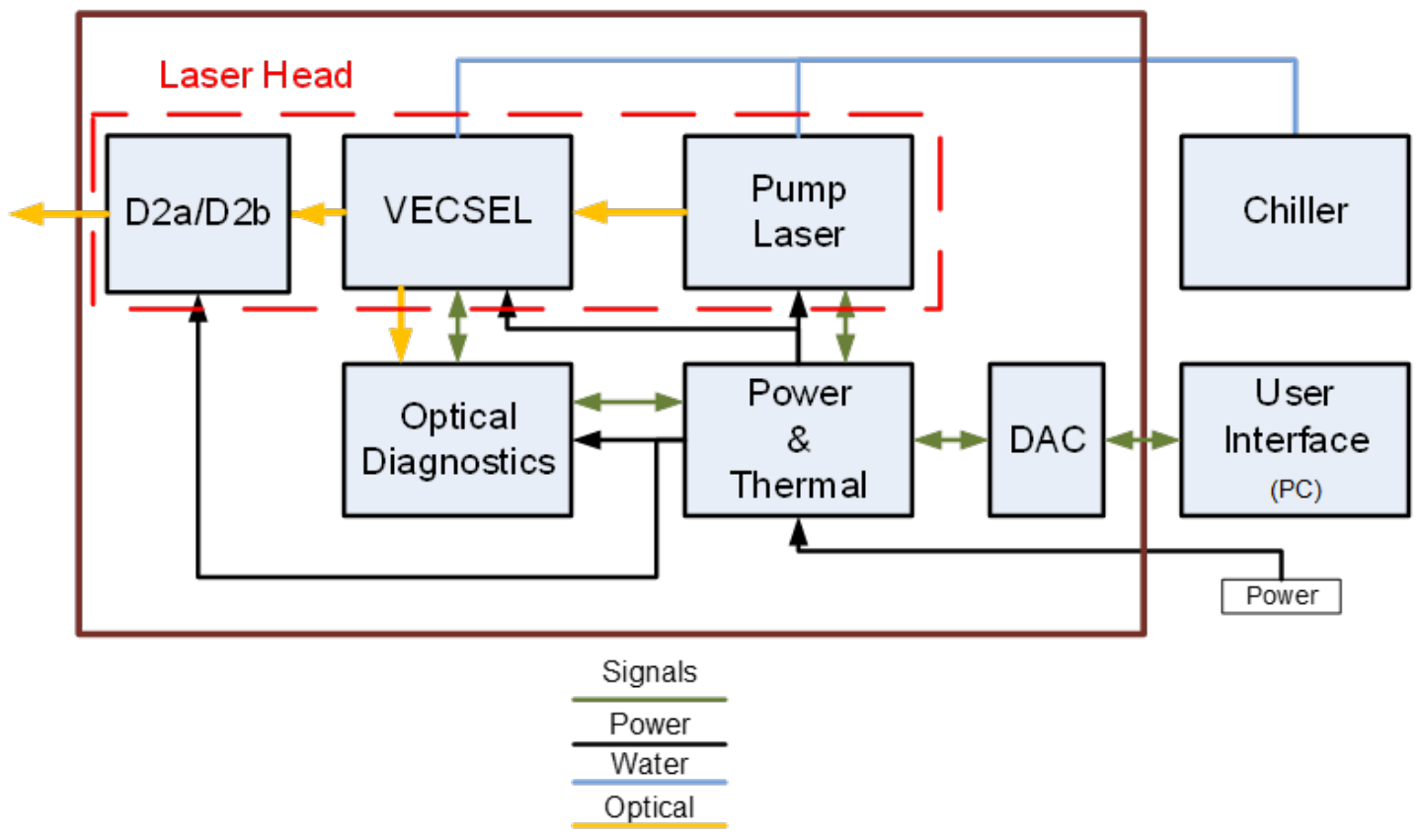

Figure 9: Schematic diagram of the Semiconductor Guidestar Laser prototype. The diagram shows the major subsystems of the laser.

\section{CONCLUSION}

This paper presents a snapshot of the Semiconductor Guidestar Laser development program as it stands half-way through its design and fabrication phase. Delivery of a robust, efficient, low SWaP prototype is expected in March 2019 at Mount Stromlo Observatory in Australia. The laser will be tested in real operating conditions on the EOS $1.8 \mathrm{~m}$ telescope in the context of Adaptive Optics imaging of satellites and space debris at Low Earth Orbit (LEO) and Geo-stationary Orbits (GEO) for the Space Environment Management Centre (SERC) ${ }^{6-8}$. ANU and their SERC partners are also planning to demonstrate maneuvering of space debris via photon pressure from a $10-20 \mathrm{~kW}$ continuous-wave infra-red laser whose beam will be pre-compensated with sodium laser guidestar adaptive optics 9 . It is expected that the Semiconductor Guidestar Laser will play an important role in these experiments and thus demonstrate its potential as a $4^{\text {th }}$ generation sodium guidestar laser for applications in astronomy, space surveillance and laser communications from the ground.

\section{ACKNOWLEDGEMENTS}

The work presented in this paper is supported via funding from ARC LIEF grant LE170100004 and ANU MEC grant 17MEC35. 


\section{REFERENCES}

[1] D'Orgeville, C., and Fetzer, G. J., "Four generations of sodium laser guide star for adaptive optics applications in astronomy and space situational awareness," 99090R, Proc. SPIE 9909 (2016)

[2] https://www.toptica.com/products/customized-solutions/sodiumstar/

[3] Holzlohner, R., Rochester, S., Calia, D. B., and Budker, D., "Optimization of cw sodium laser guide star efficiency," $A \& A 510, \mathrm{~A} 20$ (2010).

[4] Holzlöhner, R., Rochester, S. M., Pfrommer, T., Bonaccini Calia, D., Budker, D., Higbie, J. M., and Hackenberg, W., "Laser Guide Star Return Flux Simulations Based on Observed Sodium Density Profiles," Proc. SPIE 7736, 77360V (2010).

[5] D’Orgeville, C., Bouchez, A., Conan, R., Espeland, B., Gardhouse, R., Hart, J., Price, I., Trancho, G., and Uhlendorf, K., "GMT laser guide star facility," Proc. AO4ELT3 (2013).

[6] Copeland, M., Bennet, F., Rigaut, F., d'Orgeville, C., Korkiakoski, V., Smith, C., "Satellite and debris characterization in LEO and GEO using adaptive optics," Proc. AMOS conference (2017).

[7] D’Orgeville, C., Bennet, F., Blundell, M., Brister, R., Chan, A., Dawson, M., Gao, Y., Paulin, N., Price, I., Rigaut, F., Ritchie, I., Sellars, M., Smith, C., Uhlendorf, K., Wang, Y., "A sodium laser guide star facility for the ANU/EOS space debris tracking adaptive optics demonstrator," Proc. SPIE 9148, 91483E (2014).

[8] http://www.serc.org.au

[9] Lingham, M., Grosse, D., Bennet, F., Copeland, M., d’Orgeville, C., Gao, Y., Houston, E., Korkiakoski, V., Price, I., Rees, E., Rigaut, F., Smith, C., Wang., Y, "Satellite tracking with adaptive optics tracking and pushing system for space debris manoeuvre", SPIE Proc. 10703, 10703-24 (2018). 